\title{
Basal Gastric Acid level in Patients of Chronic Kidney Disease
}

\author{
Nilima Jafrin ${ }^{1}$, Nayeema Akter ${ }^{2}$, Shahin Akhter ${ }^{3}$, Md Myenuddin Mozumder \\ Sakhawat Mahmud Khan ${ }^{5}$
}

\begin{abstract}
Background: Gastrointestinal symptoms are common in patients with chronic kidney disease (CKD). Chronic renal failure is associated with an increased incidence of acid-related gastrointestinal disorders. Objective: To determine the $\mathrm{pH}$ level of gastric acid in patients of CKD and to correlate the different stages of CKD with gastric acid status. Methods: This study was carried out in the Department of Physiology and Nephrology, Chittagong Medical College and Hospital during January 2008 to December 2008. Total 87 subjects were included in this study, of them 65 patients of diagnosed case of chronic kidney diseases were selected as study group. Age and sex matched 22 apparently healthy persons were selected as control group To assess gastric juice media gastric juice $\mathrm{pH}$ level was determined with the help of Model pHs - $25 \mathrm{pH}$ meter. Data were analyzed by Chi-square test, Student's t-test and Pearson's correlation co-efficient test where applicable. Results: Mean gastric juice $\mathrm{pH}$ level of CKD patients was lower than that of control group but it was not statistically significant. Again, $\mathrm{pH}$ level of gastric juice was significantly $(\mathrm{p}<0.001)$ decreased according to severity of CKD patients. Gradual decrease of $\mathrm{pH}$ level was found with the increasing severity of the CKD. Conclusion: In this study gastric juice $\mathrm{pH}$ was lowered in patients of chronic kidney disease which was significantly lowered in stage V. So it can be concluded that the gastric acid secretion may increase in chronic renal failure patient.
\end{abstract}

Key words: Chronic kidney disease, Gastric juice $\mathrm{pH}$.

J Bangladesh Soc Physiol. 2017, June; 12(1): 10-14 For Authors Affiliation, see end of text.

http://www.banglajol.info/index.php/JBSP

\section{Introduction}

$\mathbf{G}$ astrointestinal symptoms are common in patients with chronic kidney disease (CKD), especially symptoms from upper gastrointestinal tract such as nausea, vomiting, abdominal pain, feeling of indigestion, early satiety, postprandial fullness and constipation ${ }^{1}$. Chronic renal failure (CRF) with hypergastrinemia was associated with increased stomach weight and parietal cell and enterochromaffin-like (ECL) cell density ${ }^{2}$. Chronic renal failure increases gastric mucosal blood flow and acid secretion.

Received 20 Jan 2017; $\quad$ Accepted 10 May 2017
The gastric acid secretory and mucosal hyperemic responses to pentagastrin were markedly enhanced in renal failure ${ }^{3}$. Patient develops hypertension, protienuria, progressive azotemia, and histopathological changes like focal glomerulosclerosis in chronic renal failure ${ }^{4}$. Peptic ulcer disease (PUD) has two major causes: Helicobacter pylori infection and the use of non steroidal anti-inflammatory drugs (NSAIDs). In CRF patients the cause of PUD is not an increased exposure to NSAIDs but infection of the gastric mucosa with Helicobacter pylori theoretically be expected to be increased in CKD patients ${ }^{5}$. Each day the body normally produces

J Bangladesh Soc Physiol. 2017, June; 12(1): 10-14 
about 50 to 80 millimoles more metabolic acid than metabolic alkali. The primary mechanism for removal of these acids from the body is renal excretion ${ }^{6}$. Chronic renal failure results from progressive and irreversible loss of large numbers of functioning nephrons. When kidney function declines markedly, there is a buildup of the anions of weak acids in the body fluids that are not being excreted by the kidneys. In addition, the decreased glomerular filtration rate reduces the excretion of phosphates and $\mathrm{NH}_{4}{ }^{+}$, which reduces the amount of bicarbonate added back to the body fluids. Thus, chronic renal failure can be associated with severe metabolic acidosis $^{6}$. It is to be hypothesized that chronic kidney disease is associated with an increased incidence of acid-related gastrointestinal disorders. Those disorders may be of significant concern, because the symptoms with which they are associated can result in altered dietary intake or refusal to eat, leading to malnutrition and hence, a worse prognosis. Some authors found $\mathrm{pH}$ of gastric acid was decreased in CKD patients ${ }^{7,8,9}$. Some researcher observed hyposecretion of gastric acid in some patients and hyper secretion in some patients ${ }^{\mathbf{1 0}}$. The results of the other researchers are not equivocal. Therefore, this study was undertaken to observe basal gastric acid status in chronic renal failure patients and to correlate the different stages of CKD with gastric acid status. The result of this study may help in modification of treatment procedure of chronic renal failure patients leading to good prognosis of the disease.

\section{Methods}

This cross sectional study was done in Department of Physiology, Chittagong Medical College and Department of Nephrology, Chittagong Medical College Hospital during January 2008 to December 2008. Total 87 subjects were included in this study. Sixty five patients of diagnosed case of CKD (Serum creatinine level 2 $\mathrm{mg} / \mathrm{dl}$ and above) with no anti-ulcer drugs for 2448 hours prior to the sample collection were included as study group. Age and sex matched 22 apparently healthy persons were selected as control group to compare with study group. CKD with other known preexisting diseases like bronchial asthma, liver disease; CKD with previous haematemesis and melaena, bleeding disorders e.g. Hemophilia, purpura were excluded from this study. The study protocol was approved by the ethical review committee of Chittagong Medical College.

After selection of the subjects detailed family history, medical history and physical examinations were performed and all the information were recorded on a pre-tested data collection sheet. 2- $3 \mathrm{ml}$ of blood was collected from antecubital vein to determine serum creatinine level. For collection of gastric juice the subject was asked to remain in fasting over night ( $8-10 \mathrm{hrs})$. The detailed procedure was explained to the subject to ensure his /her maximum cooperation in the morning within 7-8 am. Measurement of the tube from mouth to earlobe and down to anterior abdomen was done so that last hole on tube is below xiphoid process. This marks the distance where the tube should be inserted. Lubricant (xylocane jelly) was applied liberally to tube. Subject was asked to flex neck and tube was inserted gently into a subject naris. N-G tube was advanced into pharynx aiming posteriorly, asking the subject to swallow if possible. Once the tube has been swallowed, the subject clear speaking and breathing without difficulty was confirmed and the tube was further advanced to estimated length. Correct placement was confirmed by injecting approximately $20 \mathrm{ml}$ of air with $50 \mathrm{cc}$ syringe while auscultating epigastric area. Adhesive tape was used to tape the tube to patient's nose ensuring that pressure is not applied by tube against naris. About 5-10 $\mathrm{ml}$ of gastric juice was collected by $50 \mathrm{cc}$ syringe. 
$\mathrm{N}-\mathrm{G}$ tube was removed gently by bending it outside the nares. Gastric juice $\mathrm{pH}$ was determined with the help of Model $\mathrm{pHs}-25 \mathrm{pH}$ meter. GFR was calculated using Cockcroft and Gault formula ${ }^{11}$. Then staging of CKD patients were done by the result of this calculation. Test of significance was done by Chi-square test $\left(\chi^{2}\right)$ and Student's t-test. And to find out the correlation Pearson's correlation test was done. Data were expressed as mean \pm SD. Confidence level was fixed at $95 \%$ level and 'p' value of 0.05 or less was considered significant. Data was processed and analyzed by computer based soft ware SPSS (Statistical Package of Social Sciences) for windows version 12 .

Stages of chronic kidney disease $(\mathrm{CKD})^{12}$ :

Stage I : Normal or elevated GFR $\geq 90 \mathrm{ml} / \mathrm{min} /$ $1.73 \mathrm{~m}^{2}$

Stage II : Mild decrease in GFR $60-89 \mathrm{ml} /$ $\mathrm{min} / 1.73 \mathrm{~m}^{2}$

Stage III : Moderate decrease in GFR 30 - 59 $\mathrm{ml} / \mathrm{min} / 1.73 \mathrm{~m}^{2}$

Stage IV : Severe decrease in GFR $15-29 \mathrm{ml} /$ $\min / 1.73 \mathrm{~m}^{2}$

Stage V : Require dialysis $\leq 15 \mathrm{ml} / \mathrm{min} / 1.73 \mathrm{~m}^{2}$

\section{Results}

Mean $( \pm \mathrm{SE})$ gastric juice $\mathrm{pH}$ of $\mathrm{CKD}$ and control group were not significantly different $(>0.05)$ (Figure 1). Among CKD patients, there were none of stage I and stage II, but $8 \%$ were in stage-III, $39.1 \%$ were in stage-IV, $27.6 \%$ were in stage- $\mathrm{V}$ and $100 \%$ control group were normal. (Table I) The difference was statistically significant $(\mathrm{p}<0.001)$. Mean $\pm \mathrm{SE} \mathrm{pH}$ of gastric juice in $)$ stage III, stage IV, stage V were decreased consistently in CKD (Figure 2). $\mathrm{pH}$ of gastric juice was positively correlated with glomerular filtration rate though it was not statistically significant $(\mathrm{p}>0.05)$ (Figure 3).

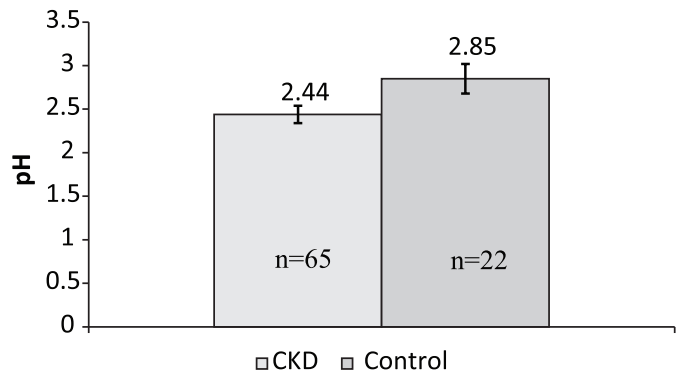

Figure 1: Gastric acid status of both groups $(\mathrm{n}=87) . \mathrm{CKD}=$ Chronic kidney disease

Table I Distribution of all subjects by GFR grading in both the groups ( $\mathrm{n}=87)$.

\begin{tabular}{lccccc}
\hline GFR & \multicolumn{2}{c}{$C K D(n=65)$} & \multicolumn{2}{l}{ Control(n=22) } \\
\cline { 2 - 3 } \cline { 5 - 6 } Grading & No. & $\%$ & & No. & $\%$ \\
\hline Stage I & 0 & 0 & 22 & 100 \\
Stage II & 0 & 0 & 0 & 0 \\
Stage III & 07 & $10.8^{* * *}$ & 0 & 0 \\
Stage IV & 34 & $52.3^{* * *}$ & 0 & 0 \\
Stage V & 24 & $36.9^{* * *}$ & 0 & 0 \\
\hline p values were obtained by $\chi^{2}$ test. $^{* * *}=\mathrm{p}<0.001$.
\end{tabular}

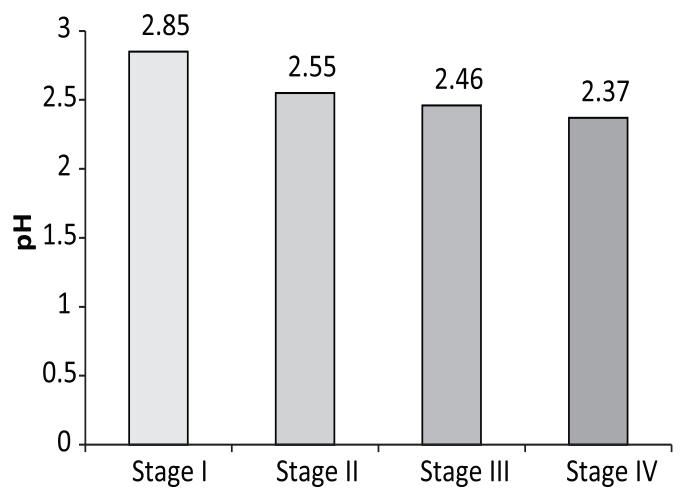

Figure 2: Mean gastric juice $\mathrm{pH}$ according to GFR grading among study groups. Stage I: GFR $\geq 90 \mathrm{ml} / \mathrm{min}$. Stage III: GFR $=30-59 \mathrm{ml} / \mathrm{min}$. Stage IV: GFR $=15-29 \mathrm{ml} / \mathrm{min}$. Stage V: GFR $\geq 15 \mathrm{ml} / \mathrm{min}$. $(n=87)$.

J Bangladesh Soc Physiol. 2017, June; 12(1): 10-14 


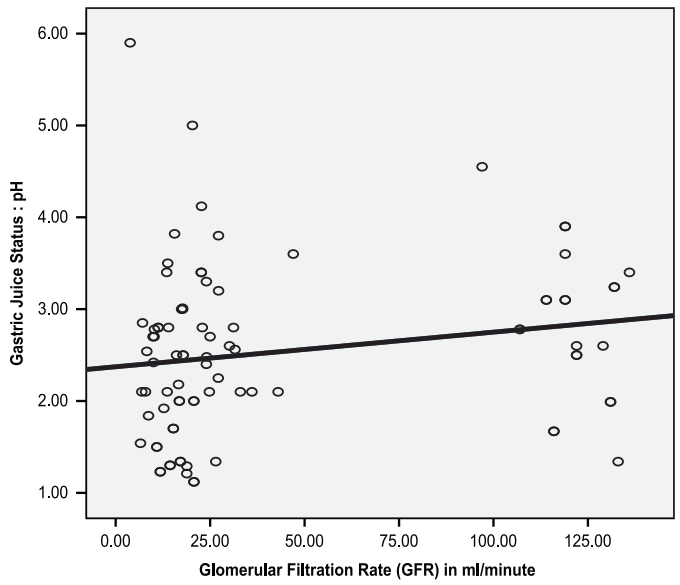

Figure 3: Correlation of Gastric juice $\mathrm{p}^{\mathrm{H}}$ with glomerular filtration rate. $(\mathrm{n}=87)$ Positive correlation was found between $\mathrm{pH}$ of gastric juice and GFR both in patients and control but not significant.

\section{Discussion}

The present study was undertaken to determine basal gastric acid status in CRF patients and to correlate the different stages of CRF with gastric acid status.

In this study, though mean gastric juice $\mathrm{pH}$ of CKD patient was lower than that of control group but this result was not statistically significant. This finding is similar with those of some other researchers ${ }^{7,8}$. Some researchers found that $26 \%$ patients with CKD had hypo secretion of gastric acid and $11 \%$ patients had hypersecrtetion of gastric acid ${ }^{10}$. Some other investigators showed gastric acid hypo secretion though there were gastric parietal, chief and G-cell cell densities in CKD patients ${ }^{9}$.

Again, total subjects were distributed according to GFR grading. Stage I considered as normal or control was $100 \%$. Among the cases there were no subjects founds in stage I \& II, Stage III was $10.8 \%$, Stage IV $52.3 \%$, Stage V $36.9 \%$. Other researchers found $45.7 \%, 50.6 \%, 3.3 \%, 0.3 \%$, $0.08 \%$ in stage I, II, III, IV, V respectively ${ }^{13}$

In our study, $\mathrm{pH}$ level of gastric juice was gradually decreased according to severity of
CKD patients. This results are similar with the findings of some other researchers where gastric hyper secretion was found in end stage renal diseases $^{3,14,15}$. Again, $\mathrm{pH}$ of gastrin juice was positively correlated with glomerular filtration rate. This finding is similar with the study of AlaKaila, K.et al ${ }^{16}$.

It has been suggested that higher acid production in CRF may be due to secondary to hypergastrinemia, which itself can be a consequence of decreased clearance of gastrin owing to a reduced glomerular filtration rate (GFR). Elevated serum gastrin level may cause an increase in acid production by the parietal cells $\mathrm{s}^{2,16}$.

\section{Conclusion}

In the present study, gastric juice $\mathrm{pH}$ was lowered in patients of chronic kidney disease which was significantly lowered in stage V. So it can be concluded that the gastric acid secretion may increase in chronic renal failure patients.

\section{Author's affiliations}

1. *Nilima Jafrin, Registrar,Bangladesh Institute of Tropical \& Infectious Diseases, Fouzderhat, Chittagong.

2. Nayeema Akter Professor \& Head (Former) Department of Physiology, Chittagong Medical College, Chittagong

3. Shahin Akhter, Assistant Professor, Department of Physiology, Chittagong Medical College, Chittagong

4. Md Myenuddin Mozumder, Junior Consultant,Orthosurgery,Chittagong General Hospital,Chittagong

5. Sakhawat Mahmud Khan ,Assistant Professor, Department of Urology, Chittagong Medical College, Chittagong

*For correspondance

\section{Conflict of interest: None}

\section{References}

1. Strid, H, Simren M, Stotzer, PO, Abrahamsson, ES. Delay in gastric emptying in patients with chronic renal failure. Scand J Gastroenterol. 2004; 6: 516520.

2. Quintero E, Ohning GV, Rivero, MD. Gastrin mediates the increase in gastric cell growth in uremic rats. Am J physiol gastrointestinal \& liver Physiology. 1995; 268: 586-591. 
3. Quintero E, Guth PH. Renal failure increases gastric mucosal blood flow and acid secretion in rats: role of endothelium-derived nitric oxide. Am J Physiol 1992; 263:75-81.

4. Ventkateswaran PS, Jeffers A, Hocken, AG. Gastric acid secretion in chronic renal failure. Brit Med Jl. 1972; 4:22-23.

5. Fallone CA, Mayrand S. Gastroesophageal reflux and hyperacidity in chronic renal failure. Peritoneal Dialysis Int. 2001; 21(3): 295-299.

6. Guyton AC, Hall JE. Urine formation by the kidneys: glomerular filtration, renal blood flow and their control. In: Text Book of Medical Physiology. $11^{\text {th }}$ ed. WB Saunders, Philadelphia; 2006. p 307.

7. Tokushima H, Tamura H, Murakawa M, Matsumur O, Itakura Y, Itoyama S, Mitarai, T, Isoda K. Eradication of Helicobacter pylori restore elevation of serum gastrin concentrations in patients with end stage renal disease. Int Med 1998; 37(5): 435439 .

8. Paimela H, Markonen, M, Koronen SL, Tallgren LG, Stenman S, Ahonen J. Relation between serum group II pepsinogen concentration and degree of brunner's gland hyperplasia in patients with chronic renal failure. Gut. 1985; 26: 198-202.

9. Paronen I, Ala-Kaila K, Rantala I, Kainulainen H, Karvonen L. Gastric parietal, chief and G-cell densities in Chronic renal failure. Scand J Gastroenterol 1991; 26: 696-700.
10. Paimela H. Persistance of gastric hypoacidity in uremic patients after renal transplantation. Scand J Gastroenterol. 1985; 20: 873-876.

11. Verhabe JC, Balje-Volkers, CP, Hillege HL, Dejong PE. The reliability of different formulae to predict creatinine clearance. J Intern Med 2003; 253:563573.

12. Sorecki K, Green J, Breener BM. Chronic renal failure. In Harrison's Principles of Internal Medicine. 16th ed. Mc Graw-Hill New York; 2005. p 1551-1562.

13. Teo BW, Ng ZY, Li J, Saw S, Sethi S. The choice of estimating equations for glomerular filtration rate significantly affects the prevalence of chronic kidney disease in a multi-ethnic population during health screening. Nephrology. 2009;14: 588-596.

14. Mitchell CJ, Jewell DP, Lewin MR. Gastric function and histology in chronic renal failure. J Clin Pathol 1979; 32(3): 208-213.

15. Ala-Kaila K, Pasternack A, Kataja M, Keyrilainen O, Sipponen P. Sensitivity of gastric acid secretion in patients with chronic renal failure. Scand J Gastroenterol 1987; 22(9): 1123-9.

16. Ala-kaila K, Kekki M, Paronen I, Paakkala, T. Serum gastrin in chronic renal failure: its relation to acid secretion, G - cell density, and upper gastrointestinal findings. Scand J Gastroenterol 1989; 24(8): 939-948. 